\title{
High Precision Phase-Shifting Electron Holography with Multiple Biprisms for GaN Semiconductor Devices
}

\author{
Kazuo Yamamoto ${ }^{1}$ \\ 1. Nanostructures Research Laboratory, Japan Fine Ceramics Center, Nagoya, Japan.
}

Electron holography is one of the promising techniques to quantitatively observe electric potential and/or magnetic flux distributions in functional materials [1,2], such as semiconductor devices. Phase reconstruction in the common electron holography is based on applying Fourier transformation and spatial frequency filter. The spatial resolution of the reconstructed phase image is determined from the filter size on the Fourier space, usually it corresponds to 2 or 3 interference fringe spacing in a hologram. Thus, it is necessary to record finer interference fringes to reconstruct the image with better spatial resolution. In this case, however, the fringe contrast drastically decreases because of limited coherency of electron waves and inelastic scattering in samples, so that it deteriorates the phase detection efficiency.

Phase-shifting electron holography [3,4] can overcome the above trade-off issue, that is, it improves the spatial resolution and the phase detection at the same time. First, we record many holograms whose interference fringes are shifted one after another by tilting the incident electron beam in a TEM. Second, we independently reconstruct the phase value in each pixel without Fourier transformation from the intensity variation in a series of the holograms. The spatial resolution is equal to the 1 pixel size on a specimen plane, and the phase detection efficiency generally increases with the number of holograms.

Here we applied the phase-shifting electron holography to a GaN semiconductor device. We prepared three kinds of bulk sample having $\mathrm{GaN} / \mathrm{Al}_{\mathrm{x}} \mathrm{Ga}_{1-\mathrm{x}} \mathrm{N}(\mathrm{x}=0.20,0.25,0.30)$ interfaces. It is known that 2dimensional electron gas (2DEG) layer is formed at the interface, and charge density of the $2 \mathrm{DEG}$ increases with the $\mathrm{Al}$ composition [5]. We prepared the TEM samples using a cryo-FIB system at $130 \mathrm{~K}$ to reduce the FIB damage layers as much as possible. Figures 1(a) and 1(b) show the typical TEM image and hologram around the $\mathrm{GaN} / \mathrm{Al}_{0.25} \mathrm{Ga} 0.75 \mathrm{~N}$ interface. The hologram was recorded under the double biprism lens condition in our $300 \mathrm{kV}$ holography TEM (HF3300-EH), in order to make uniform interference fringes without Fresnel fringes. The sample thickness was $250 \mathrm{~nm}$. Figure 1(c) shows the phase image reconstructed from 50 holograms with shifted fringes by the phase-shifting reconstruction method. The potential change is clearly observable in the GaN side at the interface, as indicated by an arrow. Figures 2(a) - 2(c) show the one-dimensional phase profiles across the GaN/A1 $\mathrm{Ca}_{\mathrm{x}} \mathrm{xN}(\mathrm{x}=0.20$, $0.25,0.30)$ interfaces, respectively. The spatial resolution was about $1 \mathrm{~nm}$ and the phase detection resolution was about $0.02 \mathrm{rad}$. We clearly observed the sharp potential changes due to the $2 \mathrm{DEG}$. Moreover, we can see that the height and width of the sharp potential increased with the Al composition.

In summary, we have succeeded in observing clear potential distribution in the GaN semiconductor device using phase-shifting electron holography. This technique is a powerful phase imaging method to observe high definition potential distribution in thick compound semiconductor samples in which finer interference fringes are not easily formed. It will be possible to observe low dopant regions (for example, $10^{17} / 10^{16} / 10^{15} / \mathrm{cm}^{3}$ level) in near future. 
References

[1] A. Tonomura, "Electron Holography", Springer Series in Optical Sciences, Vol. 70, (Springer, Heidelberg).

[2] E. Völkl, L. F. Allard, D. C. Joy (eds.), "Introduction to Electron Holography", (Kluwer Academic/Plenum Publishers, New York).

[3] Q. Ru, J. Endo, T. Tanji and A. Tonomura, Appl. Phys. Lett., 59 (1991) 2372.

[4] K. Yamamoto, I. Kawajiri, T. Tanji, M. Hibino and T. Hirayama, J. Electron Microsc., 49 (2000) 31.

[5] Q. Ambacher et al., J. Appl. Phys., 87 (2000) 334.

[6] The author thanks Mr. Yasunori Goto and Mr. Tomoyoshi Kushida in Toyota Motor Corporation for supplying the GaN samples and valuable discussion.
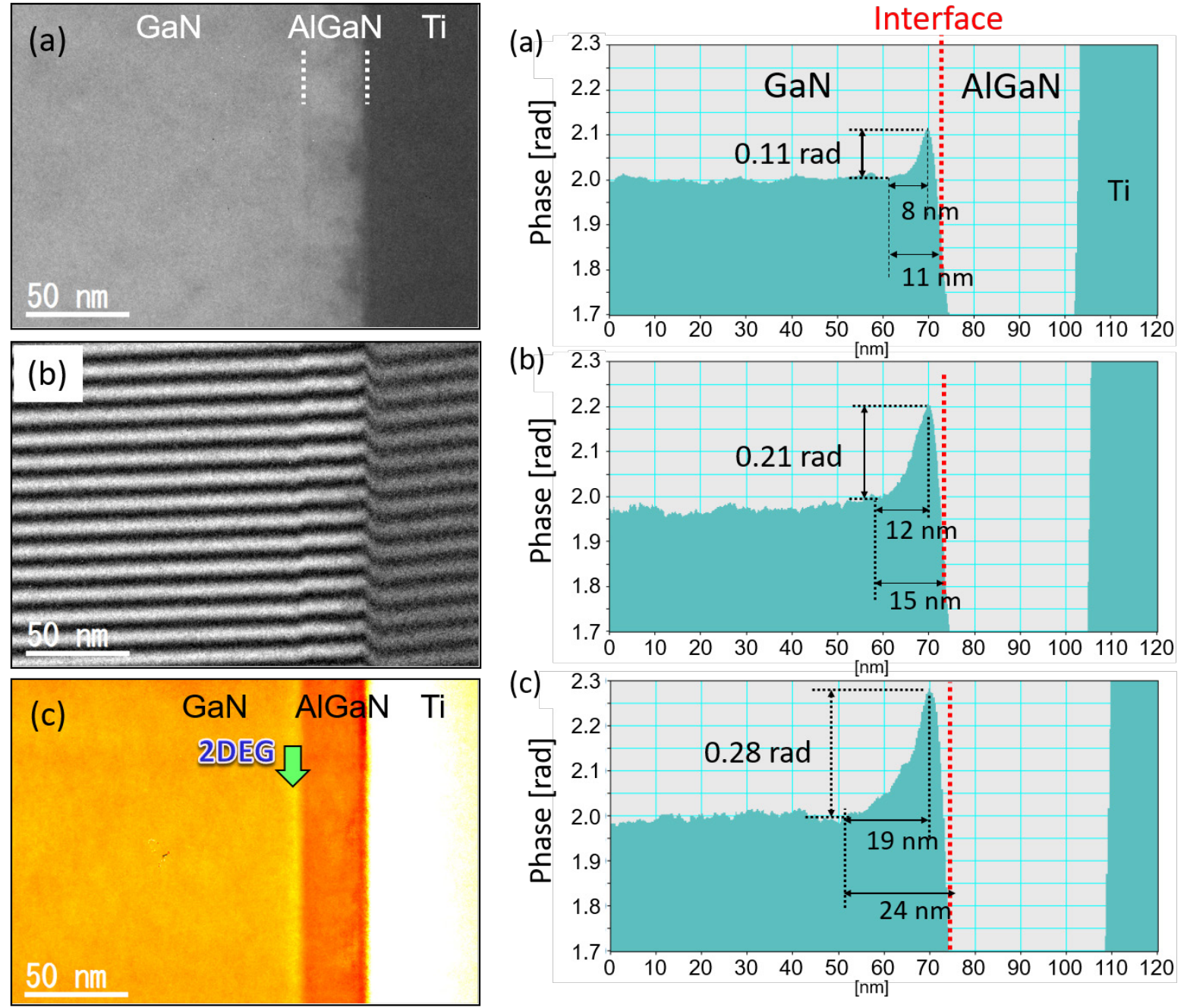

Figure 1 (a) TEM image, (b) hologram,

Figure 2 Reconstructed phase profiles across (c) reconstructed phase image of $\mathrm{GaN} / \mathrm{Al}_{0.25} \mathrm{Ga}_{0.75} \mathrm{~N}$ sample

(a) $\mathrm{GaN} / \mathrm{Al}_{0.20} \mathrm{Ga}_{0.80} \mathrm{~N}$, (b) $\mathrm{GaN} / \mathrm{Al}_{0.25} \mathrm{Ga}_{0.75} \mathrm{~N}$,

(c) $\mathrm{GaN} / \mathrm{Al}_{0.30} \mathrm{Ga}_{0.70} \mathrm{~N}$ interfaces. 